\title{
An observational cross-sectional study of the possible relationship between high levels of cytokines (TNF and ILIO) and healthcare-associated and other infections in patients with polytrauma
}

\begin{abstract}
Background: The recognition of the relationship between cytokines and outcomes in trauma patients is important. It is possible that early detection of those patients who develop an imbalance in this dual immune response, which would be responsible for subsequent organ dysfunction and susceptibility to infections, allow differential management to reduce the occurrence of such complications.
\end{abstract}

Methods: An observational cross-sectional study to describe the kinetics of cytokines/ chemokines (IL-1B, IL-6, TNF-a, IL-12p70, IL-10, and IL-8) and to explore the possible relationship with the development of infectious complications during the first admission to the hospital in patients with multiple trauma admitted to the emergency room. Serum samples were used to measured cytokines/chemokines. Times of collection were: 0-6 hour's postrauma, 24 hours and 72 hours postrauma.

Results: 20 consecutive patients were included, $60 \%$ were male, the median age was 26.5 years, $90 \%$ had a traffic accident, $40 \%$ had a severe traumatic brain injury defined as Glasgow less than or equal to 8 . The median ISS was $22(17,2-35,2)$. There was an incidence of shock defined as lactate greater than or equal to 4 of $40 \%$. The admission to ICU was $80 \%$. Of the 16 who entered the ICU, $94 \%$ required mechanical ventilation. There was only one hospital death. Cytokines shown to be significantly different between trauma patient's groups; a greater severity of the trauma in the group that presented infection (ISS 27 vs $17, p=0.011$ ). IL10 on admission and ISS are significantly associated with increased prevalence of infection in this sample of patients with multiple trauma.

Conclusion: Peripheral cytokines could present a method for tracking the course of severe blunt trauma and for better understanding this process. Our results imply that measuring cytokine expression patterns after severe blunt trauma is meaningful to explore and potentially useful in developing innovative approaches for treating acute and subacute trauma.

Keywords: blunt trauma, proinflammatory and anti-inflammatory cytokines, infections
Volume II Issue 5 - 2019

\author{
Aristizabal-Bernal Beatriz Helena, ' Vasquez \\ Edwin, ${ }^{2}$ Rincon-Caballero Olga Lucia, ${ }^{3}$ Mejía- \\ Pineda Gladys S, ${ }^{4}$ Constain-Franco Alfredo, ${ }^{5}$ \\ Jaramillo-Gomez Paula M, ${ }^{6}$ Giraldo Nelson, ${ }^{7}$ \\ Valderrama-Molina Carlos Oliver ${ }^{8}$ \\ 'Molecular Biology Coordinator, Hospital Pablo Tobon Uribe, \\ Colombia \\ MSC, Hospital Pablo Tobon Uribe, Colombia \\ ${ }^{3}$ MSC, Molecular Biology laboratory Hospital Pablo Tobon \\ Uribe, Colombia \\ ${ }^{4}$ BSC, Hematology unit, Hospital Pablo Tobon Uribe, Colombia \\ 5 MD, Trauma, Hospital Pablo Tobon Uribe, Colombia \\ MD, Surgeon, Hospital Pablo Tobon Uribe, Colombia \\ $7 \mathrm{MD}$, Intensive care, Hospital Pablo Tobon Uribe, Colombia \\ ${ }^{8}$ MD, Orthopedist, Hospital Pablo Tobon Uribe, Colombia
}

Correspondence: Beatriz H Aristizabal B, Molecular Biology Coordinator, Hospital Pablo Tobon Uribe, Colombia,Tel 5744459794,Email baristizabal@hptu.org.co

Received: September 30, 2019 | Published: October 18, 2019
Abbreviations: DOM, multiple organ dysfunctions; ARDS, acute respiratory distress syndrome; ECT, encephalo-cranial trauma; ELISA, enzyme linked immunoabsorbent assay; PICS, persistent catabolism syndrome; SIRS, systemic inflammatory response syndrome; SIRS, systemic inflammatory response syndrome; TNF-a, tumor necrosis factor alpha; IL-4, interleukin 4; IL-1, interleukin-1; AIS, abbreviated injury scale

\section{Background}

Trauma is a global public health problem, occupies one of the main places as a cause of death in the young population, below 45 years. ${ }^{1}$ Deaths in trauma have a phased behaviour, with immediate deaths due to severe head trauma or massive bleeding and late deaths usually secondary to complications. ${ }^{2,3}$

With the continuous improvement of emergency systems and access to centers specialized in trauma care, an increasing number of patients with severe trauma initially survive life-threatening injuries, but with the subsequent development of important complications such as Systemic inflammatory response syndrome (SIRS), multiple organ dysfunction (DOM) or the development of infectious complications and sepsis, with high morbidity and late mortality. ${ }^{3,4}$

Initially, severe trauma entails a local and systemic response with the production and release of inflammatory mediators that can trigger a systemic inflammatory response syndrome (SIRS) or later progress to multiple organ dysfunctions (DOM).$^{4-7}$ Also serious trauma, especially post-trauma events, such as surgical interventions (second stroke) can trigger a larger inflammatory response or a systemic antiinflammatory response (CARS) with an increased risk of infectious complications and sepsis. ${ }^{8,9}$ The greatest number of patients presents a balanced response, well tolerated, without the development of complications. ${ }^{8}$

The majority of these patients, who initially survive are young, of productive age, with a high economic and social impact. It is possible that early detection of those patients who develop an imbalance in this 
dual immune response, which would be responsible for subsequent organ dysfunction and susceptibility to infections, ${ }^{3}$ allow differential management to reduce the occurrence of such complications. ${ }^{10,11}$

The initial measurement and follow-up of different mediators (cytokines) mainly the pro-inflammatory cytokines, such as tumor necrosis factor alpha (TNF-a), interleukin-1 (IL-1), interleukin-6 (IL6) and interleukin 8 (IL-8), as well as anti-inflammatory cytokines, such as interleukin 4 (IL-4), interleukin 10 (IL-10), interleukin 13 (IL-13), as well as other mediators have been extensively studied, from the last quarter of the last century to the present, ${ }^{9,12-22}$ with some systematic reviews and meta-analyzes. ${ }^{23-26}$ In the present study, describes the pro-inflammatory response and behavior of anti-inflammatory cytokines in response to severe trauma and the possibility of developing infection and sepsis.

\section{Materials and methods}

\section{The STROBE guide is followed for the protocol, execution and report}

Design and setting: An observational cross-sectional study was performed to describe the kinematics of interleukins $1,6,8,10,12$ and tumor necrosis factor alpha (TNF a) and to explore the possible relationship with the development of infectious complications during the first admission to the hospital related to the original trauma care in patients older than 16 years with multiple trauma admitted to the emergency room of the Pablo Tobón Uribe Hospital, Medellín, Colombia, between January 2011 and December 2012.

Participants: Patients older than 16 years who had entered the emergency department of the Pablo Tobón Uribe hospital with a diagnosis of multiple trauma defined as the commitment of at least two of the corporal areas defined by the abbreviated injury scale (AIS) (head, face, thorax, abdomen, extremities or external). These patients were identified prospectively at the time of arrival at the emergency room and informed consent was obtained from the patient or his family to obtain the blood samples in order to perform the cytokine measurement at the time of admission, at 24 and at the 72 hours Patients who did not sign informed consent were excluded, as were patients with isolated brain trauma, patients admitted after 6 hours of trauma, and those who could not be sampled for the measurement of cytokines. Clinical data related to the first hospital stay were reviewed, re-admission data were not included.

Variables: In a retrospective way, the clinical records of the patients who met the inclusion criteria were reviewed. We included demographic variables such as gender, age, clinical variables at admission such as systolic blood pressure, heart and respiratory rate, lactic acid and excess of base in the first arterial gases obtained in the emergency department; the severity of the trauma was calculated according to the Injury Severity Score (ISS), the presence of brain trauma according to the Glasgow Coma Scale (ECG), the presence of shock at admission defined as systolic blood pressure less than $90 \mathrm{mmHg}$ or lactate at admission greater than $4 \mathrm{mmol} / \mathrm{L}$; Variables related to care were also measured, such as stay in critical care, need for mechanical ventilation, mortality and total hospital stay. Patients who had an infectious complication during the first hospitalization were identified. An infectious complication was defined as any infection documented in the clinical history that required antibiotic treatment based on cultures.

Measurement of cytokines: All blood samples were frozen upon collection in the emergency unit or intensive care unit (which was usually dependent on time patient presentation) then thawed once in order to aliquot into $0.5 \mathrm{ml}$ samples for storage in the laboratory at $-80^{\circ} \mathrm{C}$. All serum samples used in inflammatory biomarker inflammatory cytokines/chemokines (IL-1B, IL-6, TNF-a, IL-12p70, IL-10, and IL-8) were measured at the picogram concentration level using BDTM CBA Human Inflammatory Cytokines Kit. Times of collection were: 0-6 hour's postrauma, 24hours and 72hours postrauma.

Using the BDTM CBA Human Inflammatory Cytokines Kit can to quantitatively measure the above cytokines in serum sample. BD CBA assays provide a method of capturing a soluble analyte or set of analytes with beads of known size and fluorescence, making it possible to detect analytes using flow cytometry. Each capture bead in the kit has been conjugated with a specific antibody. The detection reagent provided in the kit is a mixture of phycoerythrin (PE)-conjugated antibodies, which provides a fluorescent signal in proportion to the amount of bound analyte. These complexes were measured using flow cytometry to identify particles with fluorescence characteristics of both the bead and the detector. The standard curve for each protein covers a defined set of concentrations from 20 to $5,000 \mathrm{pg} / \mathrm{mL}$. Analyze BD CBA Human Inflammatory Cytokines Kit data using FCAP Array software.

The normal serum levels described for the cytokines to be studied correspond to:

\section{IL1 $\beta$ : (Undetectable at $5 \mathrm{pg} / \mathrm{mL}$ )}

IL 6: (Undetectable at $4.30 \mathrm{pg} / \mathrm{mL}$ )

IL 10: (Undetectable at $13.68 \mathrm{pg} / \mathrm{mL}$ )

IL 8: $12.35 \pm 7.0 \mathrm{pg} / \mathrm{mL}$

TNF $\alpha: 6.11 \pm 3.3 \mathrm{pg} / \mathrm{mL}$

IL 12p70 (Biologically active subunit): Undetectable in sera from healthy subjects

Study size: A sample was obtained for convenience of consecutive cases that met the inclusion criteria in the study time period.

Statistical analysis: Descriptive statistics was done with relative frequencies for qualitative and median variables with inter-quartile ranges. Non-parametric means test was done to explore the differences between groups with and without infection. In addition, an exploratory multivariate analysis was performed with a logistic regression, where the dependent variable was to develop infection associated with the trauma and the interleukins, as continuous variables, and the clinical variables described in the literature were explored as independent variables.

Ethical considerations: The study had the approval from the ethics committee of research from Hospital Pablo Tobon Uribe, the procedures followed were in accordance with the ethical standards of the responsible committee on human experimentation (institutional and national) and with the Helsinki Declaration of 1975, as revised in 2013. Patients had a right to privacy that should not be violated without informed consent. Identifying information, including names, initials, or hospital numbers, are not being published.

Data archiving: All patient data were archived anonymously according to guidelines set forth by the ethics committee, and serum was frozen at $-80^{\circ}$ for future study. 


\section{Results}

20 consecutive patients were included for the description of the interleukin kinetics, $60 \%$ were male, the median age was 26.5 years (RIQ 20-35), 90\% had a traffic accident, $40 \%$ had a severe traumatic brain injury defined as Glasgow less than or equal to 8 . The median ISS was 22(17,2-35,2). There was an incidence of shock defined as lactate greater than or equal to 4 of $40 \%$. The admission to ICU was $80 \%$. Of the 16 who entered the ICU, $94 \%$ required mechanical ventilation. There was only one hospital death. Table 1 Demographic and clinical variable.

Table I Demographic and clinical variable

\begin{tabular}{lll}
\hline Qualitative variables & $\mathbf{n}$ & $\%$ \\
\hline Male & 12 & 60 \\
Traffic accident & 18 & 90 \\
Admission to ICU & 16 & 80 \\
Mechanic ventilation & 15 & 75 \\
Infection & 12 & 60 \\
TEC Serious (ECG <8) & 8 & 40 \\
Shock (SBP <90mmHg) & 4 & 20 \\
Hyperlactatemia (Lactate> 2.5mmol) & 11 & 55 \\
\hline Quantitative variable & Median & $\mathbf{P 2 5}-\mathbf{P 7 5}$ \\
\hline Age & 26.5 & $20-35,5$ \\
PAS admission & $\mathrm{II} .5$ & $101-142,2$ \\
Heart rate admission & 99 & $75-110$ \\
Respiratory frequency admission & 20 & $18-22$ \\
Admission lactate & 2.8 & $1,82-4,17$ \\
Excess base at admission & -5.7 & $(-9,5)-(-3,47)$ \\
ISS & 22 & $17,2-35,2$
\end{tabular}

ICU, Intensive care unit; TEC, brain-cranial trauma; ECG, glasgow scale; PAS, systolic blood pressure; ISS, injury severity score; P25, Percentil 25; P75, Percentil 75
12 patients $(60 \%)$ had an infection during the hospital stay, the most frequent was ventilator-associated pneumonia (5 patients), followed by peritonitis (4 patients), empyema, urinary infection and septic arthritis, one patient for each of the previous

\section{Cytokines}

Standard samples of quantitative analysis: All standard analysis of different cytokines studied was diluted from $5000 \mathrm{pg} / \mathrm{ml}$ to $0 \mathrm{pg} / \mathrm{ml}$. Cytokines shown to be significantly different between trauma patient's groups that suffer infections compared with those patients with trauma but not infections. In the bivariate analysis of the clinical variables explored and the outcome of infection, a statistically significant difference was found only with the ISS, evidencing a greater severity of the trauma in the group that presented infection (ISS 27 vs 17, $\mathrm{p}=0.011)$. In the exploration of the cytokines evaluated, statistically significant differences were found with TNFa at admission, IL10 at admission, and IL6 and IL8 at 72 hours after trauma (Table 2).

In the exploratory analysis with bivariate logistic regression, with infection outcome, it was found that IL10 on admission and ISS are significantly associated with increased prevalence of infection in this sample of patients with multiple trauma; it is possible that TNFa at admission and IL6 and IL8 at 72 hours also have association, however the small sample size of this study does not allow to reach statistical significance (Table 3).

Response of interleukins IL10 and TNF by type of infection: Testing the association between the final result of infection and the serial measurement of interleukins, the interleukins are entered into the model with an association with $\mathrm{P}$ less than 0.2 . The variables associated with the outcome independently are IL10 and high TNF $\alpha$. Patients who are prone to develop nosocomial infection have higher peaks of initial IL10 and lower levels of TNF alpha on admission.

IL-10 Normal serum concentrations in $\geq 18$ years old is (8.5$13.68 \mathrm{pg} / \mathrm{ml})$, with a media of $11.09 \mathrm{pg} / \mathrm{ml}$. Patients with trauma had increased levels of IL-10 at 0-6 hours post trauma.

Table 2 Comparison of median clinical variables and cytokines between patients with or without infection $(n=20)$

\begin{tabular}{|c|c|c|c|}
\hline Clinical variables & Infection & Non infection & \\
\hline & Median (P25 - P75) & & valor $\mathbf{p}(*)$ \\
\hline Age & $26,5(20,75-36,75)$ & $26,5(20-33)$ & 0.757 \\
\hline TAS admission & $119(101-132)$ & $122(96-1 \mid 2)$ & 0.728 \\
\hline FC admission & $99(76-116)$ & $88(59-110)$ & 0.352 \\
\hline FR admission & $20(16-23)$ & $19(18-22)$ & 0.937 \\
\hline ECG admission & $14(7-14)$ & $10(7-14)$ & 0.582 \\
\hline Lactate admission & $3,4(1,75-3,4)$ & $2,6(1,85-3,75)$ & 0.699 \\
\hline Excess Base at admission & $-5,75(-10,5--4,15)$ & $-5,75(-7,95--3,07)$ & 0.563 \\
\hline ISS & $27(22-4 I)$ & $17(13-22)$ & $0,011(* *)$ \\
\hline \multirow[t]{2}{*}{ Cytokines } & Infection & Non infection & \\
\hline & Median (P25 - P75) & & value $p(*)$ \\
\hline ILI 2 admission & - & $0(0-2,25)$ & 0.384 \\
\hline ILI $224 \mathrm{~h}$ & $0(0-0)$ & $0(0-1)$ & 0.473 \\
\hline ILI $272 \mathrm{~h}$ & $0(0-0)$ & $0(0-0)$ & 0.91 \\
\hline TNF admission & $0(0-0)$ & $1,5(0-3)$ & $0,03 \mid(* *)$ \\
\hline
\end{tabular}

Citation: Helena ABB, Edwin V, Lucia RCO, et al.An observational cross-sectional study of the possible relationship between high levels of cytokines (TNF and ILI0) and healthcare-associated and other infections in patients with polytrauma. MOJ Orthop Rheumatol. 20I9; I (5):I74-I8I. 


\begin{tabular}{|c|c|c|c|}
\hline \multirow[t]{2}{*}{ Clinical variables } & \multirow{2}{*}{$\begin{array}{l}\text { Infection } \\
\text { Median (P25 - P75) }\end{array}$} & \multicolumn{2}{|l|}{ Non infection } \\
\hline & & & valor $\mathbf{p}(*)$ \\
\hline TNF 24h & $0(0-I, 75)$ & $0(0-I, 75)$ & 0.97 \\
\hline TNF 72h & $0(0-0)$ & $0,5(0-I)$ & 0.238 \\
\hline ILI0 admission & $37,4(25,1-47,5)$ & II,3 (3,2-22,6) & $0,004(* *)$ \\
\hline ILIO 24h & $8,5(4,25-14,75)$ & $5(4-10,25)$ & 0.305 \\
\hline ILI0 72h & $4,5(3-8)$ & $3(2,25-4)$ & 0.305 \\
\hline IL6 admission & $247,5(|6|, 2-4 \mid 2,2)$ & $140,5(72-264,5)$ & 0.208 \\
\hline IL6 24h & $269,5(132-759,5)$ & $108,5(76,2-204,2)$ & 0.057 \\
\hline IL6 72h & I55 (7I,75-549) & $12(23-58)$ & $0,025(* *)$ \\
\hline ILI admission & $0(0-0,75)$ & I $(0,25-I, 75)$ & 0.057 \\
\hline ILI $24 \mathrm{~h}$ & $I(0-I)$ & $0(0-I)$ & 0.343 \\
\hline ILI 72h & $0(0-I)$ & $0(0-0,75)$ & 0.734 \\
\hline IL8 admission & $41,5(28,5-67,2)$ & $31,5(16,5-47)$ & 0.115 \\
\hline IL8 24h & $107(54,5-257,2)$ & $36(30,5-111,25)$ & 0.082 \\
\hline IL8 72h & $69(34,5-126)$ & $28(|8-4|, 5)$ & $0,0 \mathrm{I}(* *)$ \\
\hline
\end{tabular}

(*), P value for Mann Whitney test with two tails significance; (**), P<0,05 (admission to exploratory logistic regression); PAS, systolic blood pressure; FC, heart rate; FR, breathing frequency; ECG, glasgow coma scale; ISS, injury severity score; ILI 2, Interleukin I2);TNF, tumor necrosis factor alpha); ILI0, Interleukin I0; IL6, Interleukin 6; ILI, Interleukin I; IL8, Interleukin 8; P25, Percentil 25; P75, Percentil 75

Table 3 Bivariate logistic regression (Variable outcome: Infection)

\begin{tabular}{llll}
\hline Variable & OR & IC 95\% & P value \\
\hline TNF admission & 0.2384 & $0,050-$ I,II5 & 0.069 \\
ILI0 admission & I.II4 & I,0I4-I,224 & $0,024\left(^{*}\right)$ \\
IL6 72h & I.0I & $0,996-I, 024$ & 0.179 \\
IL8 72h & I.078 & $0,994-I, I 68$ & 0.07 \\
ISS & I.I72 & I,004-I,370 & $0,045\left(^{*}\right)$
\end{tabular}

OR, Odds ratio; IC 95\%, confidence interval 95\%; (*), P<0,05; TNF, tumor necrosis factor alpha; ILI0, interleukin I0; IL6, interleukin 6; IL8, interleukin 8; ISS, injury severity score

IL-6 Normal serum concentrations in $\geq 18$ years old is $(0.0-4.30 \mathrm{pg} /$ $\mathrm{ml}$ ), with a media of $2.15 \mathrm{pg} / \mathrm{ml}$. Levels of IL-6 were high throughout the study with a greater increase at 24 hours post trauma.

IL-8 Normal serum concentrations in $\geq 18$ years old are $0.0-.5 \mathrm{pg} /$ $\mathrm{ml}$, with a media of $2.5 \mathrm{pg} / \mathrm{ml}$. IL-8 levels increased significantly at 24 hours post trauma.

TNF- $\alpha$ Normal serum concentrations in $\geq 18$ years old is $6.11 \pm 3.3$ $\mathrm{pg} / \mathrm{ml}$. In the study, there were no variations in the concentrations of TNF- $\alpha$. It was found a specific patient (D026) that has higher levels of TNF- $\alpha(14.49 \mathrm{pg} / \mathrm{ml})$ at 0 hours post trauma.

IL-12p70 Normal serum concentrations in $\geq 18$ years old is no detectable, Levels of IL-12p70 in the study were in normal concentrations.
IL-1B Normal serum concentrations in $\geq 18$ years old is $(0.0-5.0 \mathrm{pg} /$ $\mathrm{ml}$ ), with a media of $2.5 \mathrm{pg} / \mathrm{ml}$. Levels of IL-1B were in normal range during the study.

\section{Discussion}

Severe trauma induces a strong immune response, which involves multiple inflammatory mediators (TNF- $\alpha$, IL- $1 \beta$, IL- 6 , IL- 8 and IL12), as well as anti-inflammatory (IL-4, IL-10, IL- 13). ${ }^{3-5}$ An imbalance between these responses would be responsible for the development of organic dysfunction, and to favor infections and sepsis. ${ }^{3-7}$ A prolonged increase in some inflammatory mediators has been shown in multiple studies as markers of adverse clinical outcome., ${ }^{913-17,19-26}$ There are several scales to establish the severity of the trauma but the ISS is one of the best associated with death and development of complications. ${ }^{27}$ Only a couple of patients in the group that did not have an infection presented a score lower than 16 , which is considered the cut-off point to define severe trauma or polytrauma, ${ }^{28,29}$ while in the patients who presented infection, all had ISS scores greater than 22 and several of them above 30 , a cut-off point that some authors refer to as a very serious trauma. ${ }^{16,28,29}$ These findings are similar to those of other studies where severe trauma is presented as one of the factors related to the development of infectious complications. ${ }^{19,30-32}$

The first proinflammatory cytokines released are TNF-a and IL-1 that act synergistically; but their presence for a very short time after the trauma, especially IL-1, makes it difficult to measure them, which is why they have not been considered clinically useful in the post-traumatic clinical setting. ${ }^{4,9,23,25}$ Contrary to our results, where the levels of IL-1 were almost undetectable and without difference in the two groups of patients, one study showed a correlation of its initial increase with the development of sepsis..$^{15}$ Regarding TNF-a, it was found elevated in the first 4 hours post-trauma ${ }^{33}$ but neither this initial increase nor its increase maintained in the following 24 
hours has been correlated with the development of complications. ${ }^{18}$ This initial increase in TNF-a was evident in our patients who did not become infected, unlike the finding of undetectable levels in those patients who later developed infection. This behavior, we could not explain, although it could indicate an inadequate initial activation of the inflammatory response (Figure 1).
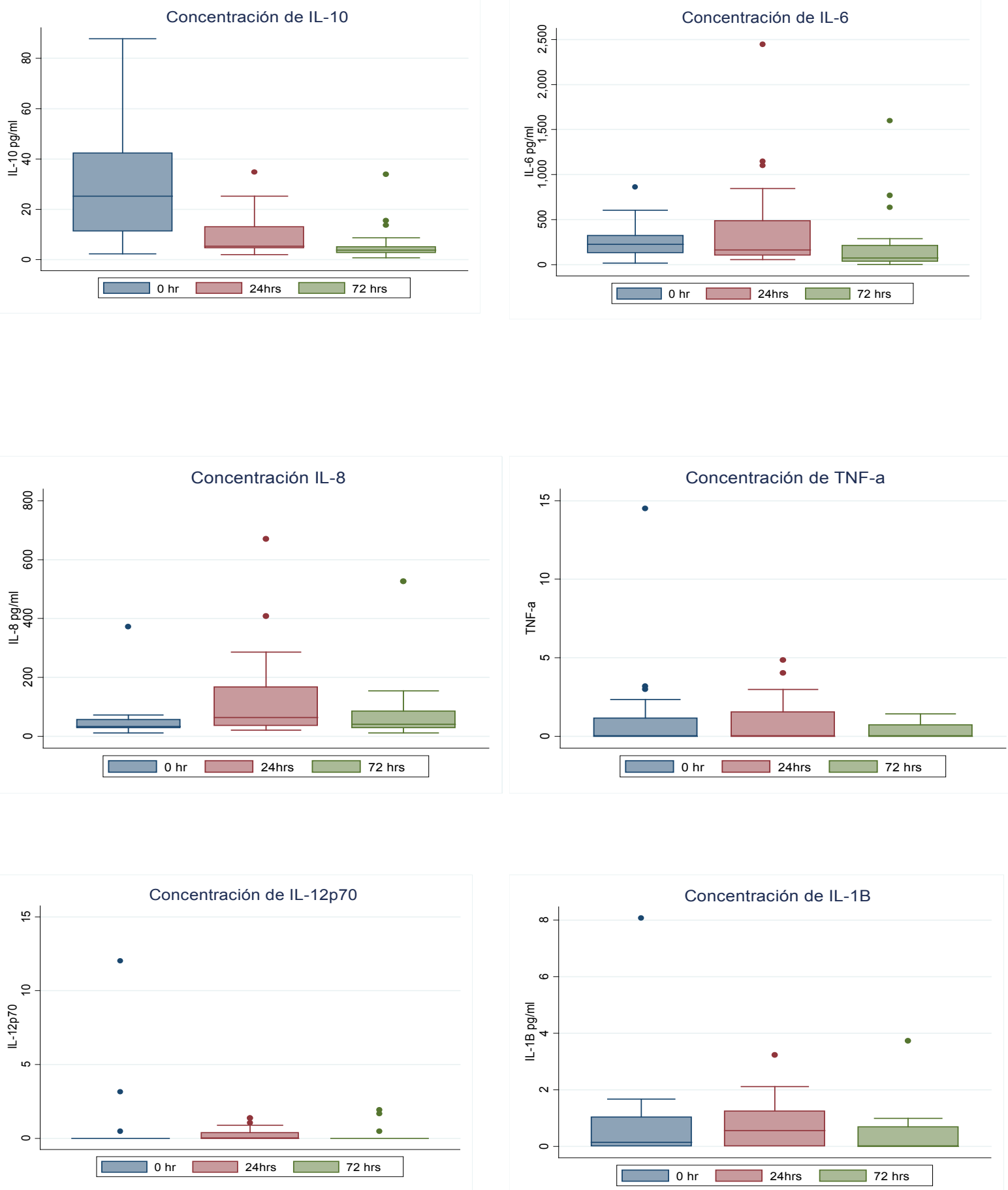

Figure I Cytokine variations on the study at 0,24 and 72 hours.

Citation: Helena ABB, Edwin V, Lucia RCO, et al.An observational cross-sectional study of the possible relationship between high levels of cytokines (TNF and ILIO) and healthcare-associated and other infections in patients with polytrauma. MOJ Orthop Rheumatol. 20 I9; I (5): I74-I8I. 
Subsequently, IL-6, one of the main cytokines of the inflammatory cascade, ${ }^{3-7}$ is released in a more sustained manner, which has been shown in several works associated with both the magnitude of the trauma and the prediction of the development of complications and death. ${ }^{9} 10,12-14,16,17,19-26$ However, the relationship between their increased levels and the development of infection is not clear in most studies, ${ }^{23-26,34-37}$ although two reports ${ }^{39,40}$ did show a relationship similar to that observed in our study. All our patients had an increase in IL-6 levels from the first measurement, but in the group that suffered infection the levels were always higher, with the maximum peak at 24 hours, however the difference was only statistically at 72 hours, unlike the work of Gouel-Chéron et al. ${ }^{38}$ where at all times the levels were significantly higher in those who later became infected, but similar to that reported by Billeter et al. ${ }^{39}$ who only showed difference from 72 hours in the group that developed sepsis. It should be noted that IL-6 also fulfills anti-inflammatory functions, especially when it is released in the subacute period of trauma. ${ }^{16,19}$ This could support, as well as the increase in IL-10, the notion of an immunoparalysis phenomenon that would explain the development of infection in our patients with significantly higher levels at 72 hours, compared to the uninfected. It is important to emphasize that a single isolated measurement of IL-6 at admission, which is usually elevated according to the severity of the trauma, is of little diagnostic utility and would require a follow-up of its behavior, to detect alterations that could indicate early on probability of complications, including the development of infections. ${ }^{22}$

The other proinflammatory cytokine, also quite studied is IL-8. Their increased levels have been very closely related to the magnitude of the trauma, ${ }^{14}$ mortality and the development of complications,,${ }^{13,14,25}$ but like IL- 6 there has not been demonstrated a clear association with the development of sepsis..$^{23,36,37}$

In the present study, the behavior of the levels of IL- 8 was similar to that of IL- 6 , it was found high from the beginning and remained high in the three measurements made, with the highest peak at 24 hours in all the patients; but unlike that reported in previous studies ${ }^{36,37}$ if it showed a significant correlation with levels that remained increased after 72 hours in patients who subsequently became infected. This behavior of IL- 8 had been shown by Vindenes et al. ${ }^{14}$ in a small group of large burns that developed sepsis, with the presence of a second late peak of IL-8 when the infection was already present; different from the behavior reported by Kirchhoff et al. ${ }^{13}$ that show a rapid downward regulation, with a fall in the levels of IL-8 in patients who presented multiple organ dysfunction as a complication. Given the small number of patients in our sample, it cannot be concluded, but this behavior of persistently elevated IL-8 should be explored in a larger population as an indicator of the subsequent development of infectious complications.

IL-12 is considered a regulatory cytokine, acting as the main bridge between innate and adaptive immunity. ${ }^{12}$ In all of our patients, levels of IL-12 were almost undetectable during the three measurement moments, which does not allow any discrimination. This probably corresponds to the fact that almost all studies report the measurement of IL-12 from its production by monocytes obtained in traumatized patients; in all of them, there is a marked decrease in their production in the post-traumatic period, in relation to the development of complications such as infections, sepsis and acute respiratory distress syndrome (ARDS). ${ }^{12-15}$ Solo Wick et al. ${ }^{15}$ report systemic measurement with equal results, where a marked decrease in IL-12 is related to the probability of dying due to multiple organ dysfunctions (DOM).
IL-10 is the main anti-inflammatory cytokine, it is released from the beginning of the trauma as a way to balance the inflammatory response, ${ }^{3-7}$ but an exaggerated release could be responsible for an immunoparalysis and the subsequent risk of infection and sepsis. ${ }^{19}$ It has been extensively studied, initially its elevation was shown as a factor of poor prognosis in the encephalo-cranial trauma (ECT) ${ }^{31}$ but subsequently the close correlation of high levels since admission has been documented, with the magnitude of any type of trauma, whether or not they have concomitantly TEC and the subsequent development of complications such as DOM, infections and death ${ }^{15,19,23,25,28}$ and especially infections and sepsis. ${ }^{15,23}$

Although the factors that impact susceptibility to infection are known, and risk prediction scales have been designed. The presence of infections in polytraumatized patients has been related to adverse clinical outcomes, unlike the subgroup of patients in whom an infectious focus is not documented, which present a lower rate of complications.

Infectious complications are a burden for severely injured patients and occur early in the critical care stay. Severity of admission shock was predictive of infection and represents an opportunity for interventions to improve infectious outcomes. ${ }^{32}$ The incidence of infection may also have utility as an end point for clinical trials in trauma. For some years, acute phase proteins, proinflammatory cytokines, soluble adhesion molecules and procalcitonin have been evaluated as markers that help identify fungal or bacterial infections, however the number of studies that include trauma patients in the hospital context is small and the predictive value is low enough to influence clinical decisions. ${ }^{23,26,39}$

Regarding the type of technique, the performance of the ELISA (Enzyme Linked Immunoabsorbent Assay) is highly dependent on the quality of the antibodies, the manufacturer of the kit, as well as the skill and experience of the operator. The ELISA allows the measurement of a single cytokine at a time in a given aliquot of sample, which becomes limiting to measure a greater number of inflammatory molecules, especially when limited quantities of biological material are available, while matrix techniques Multiple (flow cytometry, chemiluminescence or electrochemiluminescence with flow cytometry) allow the measurement of up to 25 cytokines . The advantages of flow cytometry over the ELISA technique include: high performance, less sample volume needed, efficiency in terms of time and cost, ability to evaluate the levels of an inflammatory molecule in multiple contexts and reliably detect different proteins across a wide range of concentrations. However, the degree of correlation between both methods is good.

This work shows a close relationship between the elevated levels of IL-10 and the magnitude of the trauma, evaluated by the ISS, as previously reported, ${ }^{19}$ without a special variation with the presence of concomitant TEC, given that both groups had a similar number of patients with ECT.

The behavior of the concentration of IL-10 in our patients was similar to that observed by Stensballe et al. ${ }^{19}$ but in their study, patients who did not survive were not discriminated. The highest peak was on admission, with a progressive decrease, but always with higher levels in the group of patients who presented infection, with a significant difference in the levels measured at admission. Our work supports the findings of other groups that have shown an increase in IL-10 concentrations since admission, with the subsequent development of infections and sepsis, ${ }^{15,22,23,25}$ which suggest the development of an antiinflammatory syndrome (CARS). That carries immunosuppression. ${ }^{26}$ 
The possible coexistence and perpetuation of an increase inflammatory response (SIRS), together with a dysregulated antiinflammatory response (CARS) in patients with a torpid evolution who have multiple organ dysfunction and sepsis, has been recently discussed. $^{32}$ Inflammation, immunosuppression and persistent catabolism syndrome (PICS). ${ }^{19,26}$ In the coexist varied physiological and immunological alterations, a persistent exaggerated protein catabolism, which probably require different multimodal therapies. ${ }^{33}$ Our study only evaluates the behavior of cytokines in an early period of trauma in a small group of patients, a longer follow-up and in a larger population would be required to characterize those patients who progress in a torpid form and to determine the development of this syndrome (PICS). ${ }^{33}$

The present study has several limitations; The sample size is small in comparison with other series, however, given the economic limitations in our countries, the realization of three measurements in the first 72 hours of the trauma allowed to describe the kinetics of the measured cytokines and also to explore their association with an outcome as Post-trauma infection that causes significant morbidity and mortality in the first 28 days. Another limitation was not being able to control confounding variables that could explain the variability of the cytokines, such as the magnitude of the second blow produced by the surgeries, the invasive interventions that could cause infections associated with health care, and a controlled analysis of the time to the outcome that would allow the associations found to be explored with greater scientific rigor.

\section{Conclusion}

Peripheral cytokines could present a method for tracking the course of severe blunt trauma and for better understanding this process. Although we were not able to show the prognostic value of our methods because of the limited size of our collective, our results imply that measuring cytokine expression patterns after severe blunt trauma is meaningful to explore and potentially useful in developing innovative approaches for treating acute and subacute trauma.

Cytokines could play a promising role in the follow-up of trauma patients given the potential association with the presence of bacteremia, a well-known outcome as a predictor of morbidity and mortality in this population. However, more studies with more population are required to define a clear recommendation in the management guidelines for trauma.

Additionally, with this work we were able to initiate a valuable research field based on the flow cytometry technique, with the advantages that it represents: precision and speed of sample processing for immunological study. It seems that most cytokines in our study group demonstrate a sharp decline in concentration in the first two days which plateaus at post-trauma day 3 (IL-12p70, TNF, IL-1B) with some cytokines showing increase in levels like IL-6, IL-8, IL10 at post-trauma. This information could represent the window for intervention at post-trauma.

\section{Author contribution}

Aristizabal-Bernal $\mathrm{BH}$, literature search, study design, data collection, data analysis, data interpretation, writing and critical revision.

Vasquez E, study design, data collection, data analysis, data interpretation.

Rincon-Caballero OL, study design, data collection, data analysis, data interpretation.
Mejía-Pineda Gladys S, data analysis, data interpretation.

Constain-Franco A, literature search, study design, data collection, data analysis, data interpretation, writing and critical revision.

Jaramillo-Gomez PM, literature search, data collection and critical revision.

Giraldo Nelson, study design, data collection, data analysis, data interpretation, writing and critical revision.

Valderrama-Molina CO. literature search, study design, data collection, data analysis, data interpretation, writing and critical revision.

\section{Acknowledgments}

Special thanks to Beckton Dickinson TM for their collaboration in providing us with the commercial product BD Cytometric Bead Array (CBA) Human Inflammatory Cytokines Kit and for the technical scientific advice to carry out this research work also to Alexandra Muñoz and Astrid Mora for their help.

\section{Conflict of interest}

The authors declare no conflicts of interest.

\section{Funding}

The authors have no commercial associations or sources of support from any grant, funding source.

\section{References}

1. World Health Organization. Injuries and Violence the facts. 2014;1-20.

2. Demetriades D, Murray J, Charalambrides K, et al. Trauma fatalities time and location of hospital deaths. J Am Coll Surg. 2004;198(1):20-26.

3. Keel M, Trentz O. Pathophysiology of polytrauma. Injury. 2005;36(6):691-709.

4. DeLong WG Jr, Born CT. Cytokines in patients with polytrauma. Clin Orthop Relat Res. 2004;422:57-65.

5. Giannoudis PV, Hildebrand F, Pape HC. Inflammatory serum markers in patients with multiple trauma. J Bone Joint Surg (Br). 2004;86(3):313323.

6. Lee CC, Marill KA, Carter WA, et al. A current concept of traumainduced multiorgan failure. Ann Emerg Med. 2001;38(2):170-176.

7. Lenz A, Franklin GA, Cheadle WG. Systemic inflammation after trauma. Injury. 2007;38(12):1336-1345.

8. Lasanianos NG, Kanakaris NK, Dimitrou R, et al. Second hit phenomenon: Existing evidence of clinical implications. Injury. 2011;42(7):617-629.

9. Tschoeke SK, Hellmuth M, Hostmann A, et al. The early second hit in trauma management augments the proinflammatory immune response to multiple injuries. J Trauma. 2007;62(6):1396-1404.

10. Cuscheri J, Bulger E, Schaeffer V, et al. Earle elevation in random plasma IL-6 following severe injuries associated with development of organ failure. Shock. 2010;34(4):346-351.

11. Marik PE, Flemmer M. The immune response to surgery and trauma: Implications for treatment. J Trauma Acute Care Surg. 2012;73(4):801808

12. Hamza T, Barnett JB, Bingyun Li B. Interleukin 12 a Key Immunoregulatory Cytokine in Infection Applications. Int $\mathrm{J} \mathrm{Mol} \mathrm{Sci}$. 2010;11(3):789-806. 
13. Kirchhoff C, Biberthaler P, Mutschler WE, et al. Early down-regulation of the pro-inflammatory potential of monocytes is correlated to organ dysfunction in patients after severe multiple injury: a cohort study. Crit Care. 2009;13(3):R88.

14. Vindenes H, Ulvestad E, Bjerknes R. Increased levels of circulating interleukin- 8 in patients with large burns: relation to burn size and sepsis. J Trauma. 1995;39(4):635-640.

15. Wick M, Kollig E, Walz M, et al. Does liberation of interleukin-12 correlate with the clinical course of polytraumatized patients? Chirurg. 2000;71(9):1126-1131.

16. Gebhard F, Pfetsch H, Steinbach G, et al. Is interleukin 6 an early marker of injury severity following major trauma in humans? Arch Surg. 2000;135(3):291-295

17. Pape HC, van Griensven M, Rice J, et al. Major secondary surgery in blunt trauma patients and perioperative cytokine liberation: determination of the clinical relevance of biochemical markers. $J$ Trauma. 2001;50(6):989-1000.

18. Spielmann S, Kerner T, Ahlers O, et al. Early detection of increased tumour necrosis factor alpha (TNFalpha) and soluble TNF receptor protein plasma levels after trauma reveals associations with the clinical course. Acta Anaesthesiol Scand. 2001;45(3):364-370.

19. Stensballe J, Christiansen M, Tonnesen E, et al. The early IL-6 and IL-10 response in trauma is correlated with injury severity and mortality. Acto Anaesthesiol Scand. 2009;53(4):515-521.

20. Guisasola, MC, Ortiz A, Chanab F, et al. Early inflammatory response in polytraumatized patients: Cytokines and heat shock proteins. A pilot study. Orthop Traumatol Surg Res. 2015;101(5):607-611.

21. Sapan HB, Paturusi I, Islam AA, et al. Interleukin-6 and interleukin-10 plasma levels and mRNA expression in polytrauma patients. Chin $J$ Traumatol. 2017;20(6):318-322.

22. Binkowska AM, Michalak G, Pilip S, et al. The diagnostic value of early cytokine response in patients after major trauma-preliminary report Cent Eur J Immunol. 2018;43(1):33-41.

23. Ciriello V, Gudipati S, Stavrou PZ, et al. Biomarkers predicting sepsis in polytrauma patients: Current evidence. Injury. 2013;44(12):1680-1692.

24. Easton R, Balogh ZJ. Peri-operative changes in serum immune markers after trauma: a systematic review. Injury. 2014;45(6):934-941.

25. Dekker AB, Krijnen P, Schipper IB. Predictive value of cytokines for developing complications after polytrauma. World J Crit Care Med. 2016;5(3):187-200.

26. Qiao Z, Wang W, Yin L, et al. Using IL-6 concentrations in the firs $24 \mathrm{~h}$ following trauma to predict immunological complications and mortality in trauma patients: a meta-analysis. Eur J Trauma Emerg Surg. 2018;44(5):679-687.
27. Chang L, Jlanwei T. Expression levels of tumor necrosis factor and the corresponding receptors are correlated with trauma severity. Oncology letters. 2014;8(6):2747-2753.

28. Zeiler FA, Thelin EP, Czosnyka M, et al. Cerebrospinal Fluid and Microdialysis Cytokines in Aneurysmal Subarachnoid Hemorrhage: A Scoping Systematic Review. A Front Neurol. 2017;8:379.

29. Reikeras O, Borgen P, Reseland JE, et al. Changes in serum cytokines in response to musculoskeletal surgical trauma. BMC Res Notes. $2014 ; 7: 128$

30. Ozturk H, Yagmur Y, Ozturk H. The prognostic importance of serum IL-1beta, IL-6, IL-8 and TNF-alpha levels compared to trauma scoring systems for early mortality in children with blunt trauma. Pediatr Surg Int. 2008;24(2):235-239.

31. Kumar A, Stoica BA, Loane DJ, et al. Microglial-derived microparticles mediate neuroinflammation after traumatic brain injury. $J$ Neuroinflammation. 2017;14(1):47.

32. Gee K, Guzzo C, Che Mat NF, et al. The IL-12 Family of Cytokines in Infection, Inflammation and Autoimmune Disorders. Inflamm Allergy Drug Targets. 2009;8(1):40-52.

33. Gentile LF, Cuenca AG, Efron PA, et al. Persistent inflammation and immunosuppression: A common syndrome and new horizon for surgica intensive care. J Trauma Acute Care Surg. 2012;72(6):1491-1501.

34. Liener UC, Brückner UB, Knöferl MW, et al. Chemokine activation within 24 hours after blunt accident trauma. Shock. 2002;17(3):169-172.

35. Haasper C, Kalmbach M, Dikos GD, et al. Prognostic value of procalcitonin (PCT) and/or interleukin-6 (IL-6) plasma levels after multiple trauma for the development of multiorgan dysfunction syndrome (MODS) or sepsis. Tech Helth Care. 2010;18(2):89-100.

36. Egger G, Aigner R, Glasner A, et al. Blood polymorphonuclear leukocyte migration as a predictive marker for infections in severe trauma: comparison with various inflammation parameters. Intensive Care Med. 2004;30(2):331-334.

37. Giamarellos-Bourboulis EJ, Mouktaroudi M, Tsaganos T, et al. Evidence for the participation of soluble triggering receptor expressed on myeloid cells- 1 in the systemic inflammatory response syndrome after multiple trauma. J Trauma. 2008;65(6):1385-1390.

38. Gouel-Chéron A, Allaouchiche B, Guignant C, et al. Early interleukin-6 and slope of monocyte human leukocyte antigen-DR: a powerful association to predict the development of sepsis after major trauma. PLoSOne. 2012;7(3):e33095.

39. Billeter A, Turina M, Seifert B, et al. Early serum procalcitonin, interleukin-6, and 24-hour lactate clearance: useful indicators of septic infections in severely traumatized patients. World J Surg. 2009;33(3):558-566. 